\title{
Fast and Efficent Multimodal Eye Biometrics using Projective Dictionary Pair Learning
}

\author{
Abhijit Das $^{\mathrm{a}}$, Prabir Mondal ${ }^{\mathrm{b}}$, Umapada Pal ${ }^{\mathrm{b}}$, Miguel Angel Ferrer ${ }^{\mathrm{c}}$ and Michael Blumenstein ${ }^{\mathrm{a}}$ \\ ${ }^{a}$ Institute for Integrated and Intelligent Systems, Griffith University, Queensland, Australia \\ Email: abhijit.das@griffithuni.edu.au \\ ${ }^{\mathrm{b}}$ Computer Vision and Pattern Recognition Unit, Indian Statistical Institute, Kolkata, India, Email: umapada@isical.ac.in \\ ${ }^{\mathrm{c}}$ IDeTIC, University of Las Palmas de Gran Canaria, Las Palmas, Spain, Email: mferrer@dsc.ulpgc.es \\ ${ }^{\mathrm{d} S}$ School of Software, University of Technology Sydney, Australia, Email: michael.blumenstein@uts.edu.au
}

\begin{abstract}
This work proposes a projective pairwise dictionary learning-based approach for fast and efficient multimodal eye biometrics. The work uses a faster Projective pairwise Discriminative Dictionary Learning (DL) in contrast to the traditional DL which uses synthesis DL. Projective Pairwise Discriminative Dictionary (PPDD) uses a synthesis dictionary and an analysis dictionary jointly to achieve the goal of pattern representation and discrimination. As the PPDD process of DL is in contrast to the use of $l_{0}$ or 1 -norm sparsity constraints on the representation coefficients adopted in most traditional DL, it works faster than other DL. Moreover, the blending of synthesis dictionary and an analysis dictionary also enhance the feature representation of the complex eye patterns. We employed the combination of sclera and iris traits to establish multimodal biometrics. The experimental study and analysis conducted fulfill the hypothesis we considered. In this work we employed a part of the UBIRIS version 1 dataset to conduct the experiments.
\end{abstract}

Keywords - Sclera; Iris; Biometrics; Dictionary Learning; Synthesis Dictionary, Multimodal Biometrics.

\section{INTRODUCTION}

Biometrics is a technology that uses specific physical or biological characteristics to verify the identity of an individual. Its primary purpose is to tighten security. Biometric technology uses computerized methods to identify a person by their unique physical or behavioral characteristics. Biometrics refers to metrics related to human characteristics. Biometrics authentication (or realistic authentication) is used in computer science as a form of identification and access control. It is also used to identify individuals in groups that are under surveillance. Biometric is more secure than token-based security systems such as passport photo or pin [2]. Using traits such as fingerprints, face or eyes as key identity features, biometric models are established. Lately, along with fingerprints and face, the iris is also taken into account for biometric identification porous.

Iris recognition is an automated method of biometric identification that uses mathematical patternrecognition techniques on images of one or both of the irises of an individual's eyes, whose complex random patterns are unique, stable, and can be seen from some distance. Camera technology with subtle near infrared illumination are used in iris recognition to acquire images of the detail-rich, intricate structures of the iris, which are externally visible. A key advantage of iris recognition, besides its discernment of patterns and its extreme resistance to false matches, is the stability of the iris as an internal and protected, yet externally visible organ of the eye.

A typical iris biometric system consists of image capture, iris localization, iris pre-processing, feature extraction and pattern classification. Most of the deployed and commercially available iris recognition systems typically use Daugman's [1] algorithms for iris localization. With the advancements in the literature it is evident that the template-matching approaches used in the above techniques reduce the usability of the systems i.e. with respect to reasonable size of the database and also the time complexity of the system. Therefore, different feature extraction techniques such as 1D log-Gabor filters (LG) [3], SIFT operator (SIFT) [3], local intensity variations in iris textures (CR) [5], Discrete-Cosine Transform (DCT) [6], cumulative sum-based grey change analysis (KO) [7], and Gabor spatial filters (QSW) [8] are explored in the literature. For the classification task also, stronger classifiers such as Support Vector Machine (SVMs) and Artificial Neural Network (ANNs) are being explored to ensure lower time complexity of iris recognition and an increase in the accuracy of iris recognition.

Besides several advantages of the iris, there are also several disadvantages that restrict its universal application. For example, the capture of iris images requires the cooperation of the user since an off-axis iris image can deteriorate system performance; moreover recognition at a distance is only possible for a small range. For all these reason and the ease of using this trait in mobile environments, iris biometrics in the visible spectrum are proposed [23]. In this regards, the accuracy drops dramatically for darker irises. To overcome this problem, multimodal iris biometrics in combination with other eye traits like sclera (white part of the eye which contains the vessel patterns and can be used as a biometric trait) or peri-ocular can be used. In our experiment we considered sclera for combination as it is more promising one.

To our knowledge, the first recognized work on sclera biometrics was proposed in [21]. A survey on sclera recognition is described in [26]. It is evident from the literature 
that most of the individual work on the sclera [23, 27] and multimodal eye recognition techniques [22, 24, 25] using the sclera and the iris employs a template matching-based technique for pattern classification, which is quite time consuming. Although in subsequent work of sclera biometrics [28-32] sophisticated classifier techniques such as Support Vector Machines are employed, however the time complexity of the feature extraction process is quite high.

To deal with the aforementioned problems of complexity and efficient pattern classification, discriminative dictionary learning (DL) can be studied. The aims for most of the conventional DL methods are to learn a synthesis dictionary to represent the input signal while the representation coefficients, represent the residual to be discriminative. Adopting the " $l_{0 \text {-norm" }}$ and " $l_{1-\text { norm" }}$ sparsity constraint on the representation coefficient, the time complexity for the training and testing phases places a major disadvantage on them. The discriminative DL framework, namely projective dictionary pair learning (DPL), has been used to deal with pattern classification problems with an optimized time complexity. The DPL learns a synthesis dictionary as well as an analysis dictionary together for signal representation and discrimination. DPL does not only reduce the time complexity in the training and testing phases, but also leads a very effective and high accuracy in a variety of visual classification tasks. Inspired by the method proposed in [13], we have hypothesized to explore DPL on multimodal eye recognition for better accuracy and reduced computational time complexity for the recognition task.

The organization of the remaining part of the paper is as follows: Section II explains the proposed pattern classification approach for multimodal eye biometrics. In Section III, the experimental details are described and Section IV draws the overall conclusions.

\section{PROPOSED PATTERN CLASSIFICATION TECHNIQUE FOR MULIMODAL EYE BIOMETRICS}

In the recent literature, Dictionary Learning (DL)-based feature extraction has evolved to be one of the most promising feature extraction techniques. These techniques can also be explored for efficient biometric pattern recognition.

Studies on sparse representation with a synthesis dictionary have drawn a major impact widely in the recent years of study in $[9,10,11]$. The "lp-norm" $(p<=1)$ sparse coding which is computationally more expensive than analytical dictionarybased representation is used to obtain the representation coefficients of a pattern for the synthesis dictionary. Complex local image structures are modelled better by synthesis-based representation. This representation has led to many state-of-theart results for image recognition. Allowing us to easily learn a desired dictionary from the training data is another important advantage of the synthesis-based sparse representation. The seminal work of KSVD [12] tells us that an over-complete dictionary can be learned from example natural images, and it can lead to much better image reconstruction results than the analytically designed off-the-shelf dictionaries. Many dictionary learning (DL) methods have been proposed and state-of-the-art performance in the image recognition task has been achieved by utilizing KSVD. Regardless of these advantages, this synthesis DL poses high time complexity due

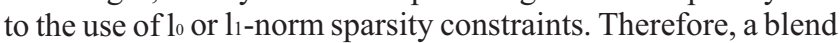
of the synthesis and analytical-based DL can be assumed to overcome the aforementioned pitfall, preserving the pattern recognition accuracy. As a reason in this work, we conceived to explore such a dictionary-based leaning for a multimodal eye pattern recognition task. We hypothesize to explore a projective dictionary pair learning for a biometric pattern recognition task. In the next subsection, discriminative dictionary learning, the projective dictionary pair learning model and the classification scheme is explained.

\section{A. Discriminative dictionary learning}

Let $X=\left[X_{I p}, X_{2 p}, \ldots \ldots, X_{K p}\right]$ be the training samples set having $\mathrm{p}$ dimensionality from $k$ classes. Where, $X_{k}$ is a p-by-n matrix or a training sample set of class $k$, considering $\mathrm{n}$ is the number of samples of each class. An effective data representation model from $X$ is learned by the discriminative DL methods for the classification tasks by exploiting the class label information in the training phase. The discriminative DL methods can be expressed as follows:

$$
\min _{D, A}\|X-D A\|_{F}^{2}+\lambda\|A\|_{p}+\psi(D, A, Y)
$$

Here $Y$ represents the class label matrix of samples in $X, \lambda$ is a scalar constant $(>=0), D$ is the synthesis dictionary that has to be learned and $A$ is the coding coefficient matrix of $X$ on $D$. Therefore in the training model, $\|X-D A\|_{F}^{2}$ ensures representation efficiency of $D,\|A\|_{p}$ represents the ' $l_{p \text {-norm }}$ " regularizer on $A$ and $\psi(D, A, Y)$ represents the discriminative function. Discriminative Learning works in two ways: some of them $[15,16,17]$ learn a structured dictionary for the discrimination of classes, whereas others $[10,11,14]$ shared a dictionary for all classes and a classifier on the coding coefficients simultaneously. The " $l_{0 \text {-norm" }}$ and " $l_{\text {-norm" }}$ " sparsity are regularizer on the coding coefficients and are applied by all the DL methods. So there is some inefficiency in the training phase and the consequent testing phase.

The formula in (1) is for the conventional DL model. It learns a discriminative synthesis dictionary. In the DPL method, we have used the DL which was extended to a novel DPL model that learns both synthesis and analysis dictionaries. It shows time computational efficiency as " $l_{0 \text {-norm" }}$ or " $l_{1 \text {-norm }}$ " and the sparsity regularizer is not required for the coding coefficients rather it can be explicitly learned by linear projection.

\section{B. Projective dictionary pair learning model}

Learning a synthesis dictionary $D$ to sparsely represent the signal $X$ is the main objective of the conventional discriminative model in equation (1). To resolve the code $\mathrm{A}$, the costly " $l_{\text {-norm" }}$ sparse coding process is required. The representation of $X$ would be very efficient if we could find an analysis dictionary $(P)$ which could satisfy $A=P X$. To do this, 
an analysis dictionary is learnt with the synthesis dictionary $D$ and the model obtained can be formulated as follows,

$$
\left\{P^{*}, D^{*}\right\}=\arg \min _{P, D}\|X-D P X\|_{F}^{2}+\psi(D, P, X, Y)
$$

Here to analytically code $X$, the analysis dictionary $P$ is implemented and the synthesis dictionary $D$ is used to reconstruct $X$. Where, $\psi(D, P, X, Y)$ is some discrimination function.

So the structured synthesis dictionary $D=\left[D_{1}, D_{2}, \ldots, D_{K}\right]$ and a structured analysis dictionary $P=\left[P_{1}, P_{2}, \ldots, P_{K}\right]$ is learned. Here $D_{k}$ and $P_{k}$ take part to produce a sub-dictionary pair corresponding to class $k$. The efficiency of the DPL model depends on the design of the discrimination function. Now the sparse subspace clustering [18] has proved that if signals satisfy certain incoherence conditions then the sample can be represented by its corresponding dictionary. It is desired that $P_{k}$ should project the samples from class $i$ (where $i$ not equal to $k$ ) towards a null space with the structured analysis dictionary P. It can be formulated as follows,

$$
P_{k} X_{i} \approx 0, \forall k \neq i
$$

Similarly, the structured synthesis dictionary $D$ can reconstruct the data matrix $X$. The data matrix $X_{k}$ can be reconstructed efficiently by the sub-directory $D_{k}$ from the projective code matrix $P_{k} X_{k}$. Hence the dictionary pair is used to minimize the reconstruction error. So,

$$
\min _{P, D} \sum_{k=1}^{k}\left\|X_{k}-D_{k} P_{k} X_{k}\right\|_{F}^{2}
$$

and according to the above discussion the DPL model can be formulated as,

$$
\begin{gathered}
\left\{P^{*}, D^{*}\right\}=\arg \min _{P, D} \sum_{k=1}^{k}\left\|X_{k}-D_{k} P_{k} X_{k}\right\|_{F}^{2}+\left.\lambda\left\|P_{k} \bar{X}_{k}\right\|\right|_{F} ^{2} \\
\text { s.t. }\left\|d_{i}\right\|_{2}^{2} \leq 1
\end{gathered}
$$

Here, $\bar{X}_{k}$ is the complement of $X_{k}$; in the whole training set $X$; the $i^{\text {th }}$ atom of synthesis dictionary $D$ is $d i$ whose energy is constrained to avoid the trivial solution $P_{k}=0$ to make the DPL stable. $\lambda$ is a scalar constant which is greater than 0 .

It is argued that sparse coding may not be crucial in classification $[19,20]$. But the DPL model is much faster, and it has very competitive classification performance. Therefore, for the classification scheme, we used the following approach.

For optimization purposes the following methodology due to [13] is used. The objective function in (5) is generally nonconvex. A variable matrix $A$ is introduced and relaxed (5) to the following problem:

$$
\left\{P^{*}, A, D^{*}\right\}=\arg \min _{P, A, D} \sum_{k=1}^{k}|| X_{k}-D_{k} A_{k} \|_{F}^{2}
$$

$$
\begin{gathered}
=|| P_{k} X_{k}-A_{k} \|\left.\right|_{F} ^{2}+\lambda|| P_{k} \bar{X}_{k}||_{F}^{2}, \\
\text { s.t. }\left\|d_{i}\right\|_{2}^{2} \leq 1
\end{gathered}
$$

where $\tau$ is a scalar constant. All terms in the above objective function are characterized by the Frobenius norm, and (6) can be easily solved. We initialize the analysis dictionary $P$ and synthesis dictionary $D$ as random matrices with the unit Frobenius norm, and then alternatively update $A$ and $\{D, P\}$. The minimization can be alternated between the following two steps.

a) Fix D and P, update A

$$
A^{*}=\arg _{A}^{\min } \sum_{k=1}^{k}\left\|X_{k}-D_{k} A_{k}\right\|_{F}^{2}+\tau\left\|P_{k} X_{k}-A_{k}\right\|_{F}^{2}
$$

This is a standard least squares problem and we have the closedform solution:

$$
A_{k}^{*}=\left(D_{k}^{T} D_{k}+\tau \mathrm{I}\right)^{-1}\left(\tau P_{k} X_{k}+D_{k}^{T} X_{k}\right)
$$

b) Fix A, update D and P:

$$
\left\{\begin{array}{c}
P^{*}=\arg _{p}^{\min } \sum_{k=1}^{k}|| P_{k} X_{k}-\left.A_{k}\left\|_{F}^{2}+\lambda|| P_{k} \bar{X}_{k}\right\|\right|_{F} ^{2} \\
D^{*}=\left.\arg { }_{D}^{\min } \sum_{k=1}^{k}\left\|X_{k}-D_{k} A_{k}\right\|\right|_{F} ^{2} \\
\text { s.t. }\left\|d_{i}\right\|_{2}^{2} \leq 1 .
\end{array}\right.
$$

The closed-form solutions of $P$ can be obtained as:

$$
P_{k}^{*}=\tau A_{k} X_{k}^{T}\left(\tau X_{k} X_{i}^{T}+\lambda \bar{X}_{k} X_{k}^{T}+\gamma I\right)
$$

where,

$\gamma=10 e-4$ is a small number.

The $D$ problem can be optimized by introducing a variable $S$ :

$$
\begin{aligned}
& \min _{D, s} \sum_{k=1}^{k}|| X_{k}-D_{k} A_{k} \|_{F}^{2} \\
& \text { s.t. } D=S, \quad\left\|S_{i}\right\|_{2}^{2} \leq 1 .
\end{aligned}
$$

The optimal solution of (11) can be obtained by the ADMM algorithm: 


$$
\left\{\begin{array}{c}
D^{(r+1)}=\arg _{D}^{\min } \sum_{k=1}^{k}\left\|X_{k}-D_{k} A_{k}\right\|_{F}^{2}+ \\
p\left\|D_{k}-S_{k}^{(r)}+T_{k}^{(r)}\right\|_{F}^{2} \\
S^{(r+1)}=\arg _{S}^{\min } \sum_{k=1}^{k} p\left\|D_{k}^{(r+1)}-S_{k}^{(r)}+T_{k}^{(r)}\right\|_{F}^{2} \\
\text { s.t. } D=\left\|S_{i}\right\|_{2}^{2} \leq 1 \\
T^{(r+1)}=T^{(r)}+D_{k}^{(r+1)}-S_{k}^{(r+1)} \\
\text { update } p \text { if appropiate }
\end{array}\right.
$$

In each step of optimization, closed form solutions for variables $A$ and $P$, and the ADMM-based optimization of $D$ converges rapidly. The training of the proposed DPL model is much faster than most previous discriminative DL methods. When the difference between the energy in two adjacent iterations is less than 0.01 , the iteration stops. The analysis dictionary $P$ and the synthesis dictionary $D$ are then output for classification. One can see that the first sub-objective function in (9) is a discriminative analysis dictionary learner, focusing on promoting the discriminative power of $P$; the second subobjective function in (9) is a representative synthesis dictionary learner, aiming to minimize the reconstruction error of the input signal with the coding coefficients generated by the analysis dictionary $P$. When the minimization process converges, a balance between the discrimination and representation power of the model can be achieved.

\section{Classification scheme}

Classification is performed based on the residual value over the samples for a class. $P_{k}(*)$ is the analysis sub-directory trained to produce small coefficients for samples from classes other than $k$ while the $D_{k}(*)$ is the synthesis sub-directory trained to reconstruct the samples of class $k$. Then the residual $\left\|X_{k}-D_{k}^{*} P_{k}^{*} X_{k}\right\|_{F}^{2}$ will be smaller than the residual $\| X_{i}-$ $D_{k}^{*} P_{k}^{*} X_{i} \|_{F}^{2}$ when $i \neq k$.

In the testing phase, a query sample y of an unknown class is considered a query image and its residual is calculated for every class. The class having the minimum residual is the class of the testing sample.

The testing is formulated as follows,

$$
\operatorname{identity}_{(y)}=\arg _{\min _{i}}\left\|y-D_{i} P_{i} y\right\|_{2}
$$

Here $D_{i}$ and $P_{i}$ are the synthesis sub-directory and the analysis sub-directory respectively for class $i$. So according to (12), a sample from say class $i$, would be the class of the sample $\mathrm{y}$ if the minimum residual is obtained from (12) for class $i$.

\section{EXPERIMENTAL RESULTS AND DISCUSSION}

The experimental results and the dataset employed for the experimentation are explained in the subsections below.

\section{A. Data Set}

Database UBIRIS Version 1 [33] has been taken as the data set in order to implement our proposed method and evaluate its performance. This database consists of 1877 RGB images taken in two distinct sessions (1205 images in session 1 and 672 images in session 2) from 241 identities where each channel of RGB colour space is represented in grey-scale. Blurred images and images with blinking eyes are also present in the data set. Images with high resolution $(800 \times 600)$ and low resolution $(200 \times 150)$ are also represented in the database. All the images are in JPEG format.

Here we considered only those classes for which images both from session 1 as well as session 2 are present (as the employed dictionary learning process performs better with an equal number of samples per class). In our method we have 127 classes and ten images from every class (5 images from session1 and five images from session2). We have used different quality images and some of the sample images are shown below in Figure 1.

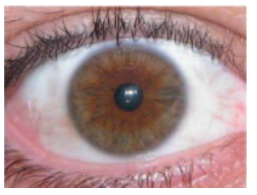

(a)

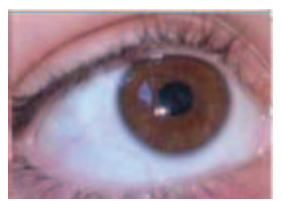

(d)

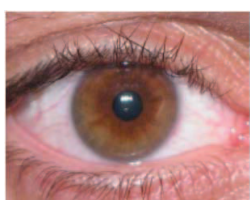

(b)

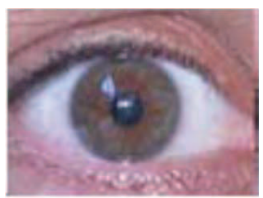

(e)

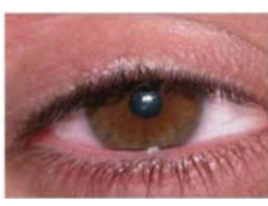

(c)

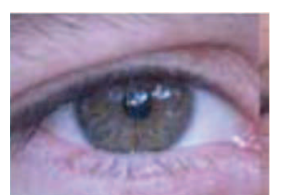

(f)
Figure 1: Different quality of eye images used in the experiments (a) is the type of best quality image of Session 1, (b) is the type of medium quality image of Session 1 (c) is the type of poor quality image of Session 1, (d) is the type of poor quality image of Session 2, (e) is the type of medium quality image of Session 2 and (f) is the type of best quality image of Session 2.

Images in the data set are different in quality. Some of them are not occluded having good quality of sclera regions. Images with medium quality and poor quality with respect to sclera region visibility are also present in the database. Some closed eye images were also taken for the experiments, and some of them are shown in Figure 2. The first session images were taken in a dark room so that the noise factors such as reflection, luminosity, and contrast were minimized. In the second session, the images were taken under natural illumination conditions with spontaneous user participation in order to introduce natural luminosity and add more noise factors than the first session. The database contains blurred images and images with blinking eyes as shown in Figure 2. All the images from session 1 and session 2 were considered in the experiments. Experiments took 
place in two categories: single-session and multi-session. In single session experiments, session 1 and session 2 were considered separately; from each class, 3 images were chosen randomly and used for training whilst the other 2 images were taken for testing the performance. From session-1, 5 images were considered for training, and the remaining 5 images from session 2 for testing.

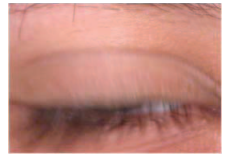

(a)

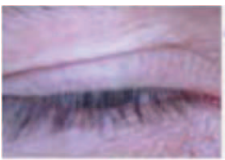

(d)

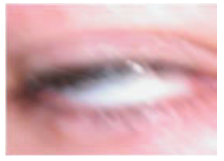

(b)

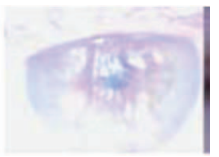

(e)

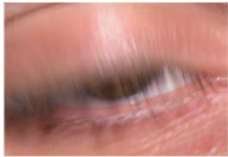

(c)

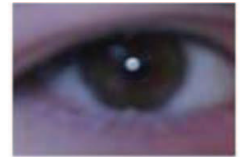

(f)
Figure 2: Examples of closed and blurred eyes. (a), (b) and (c) are from session 1 and (d), (e) and (f) are from session 2.

In our experiments, 5 images from each class were used for training and 5 for testing. Therefore, in the experiments, the considered FRR score and FAR score were $127 * 5$ and $127 * 126 * 5$ respectively.

All the simulation experiments performed here were developed in Matlab 2013a on the Windows7 operating system along with a core I5 processor and 8GB RAM as the hardware configuration.

\section{B. Image pre-processing}

For the ease of the algorithm, the images were down sampled by image resizing techniques to a different size. The down sampling was undertaken for low resolution images also.

In order to get the segmented iris, Daugmans integrodifferential method [1], was used to calculate the center of the iris. The iris image was cropped considering its radius length, along the iris center as shown in Figure 3a. This method was also able to spot the iris region perfectly for the closed eye images.

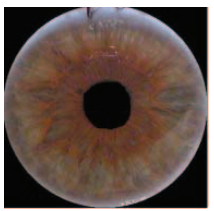

(a)

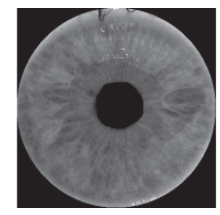

(b)

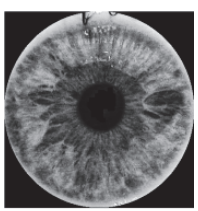

(c)
Figure 3: (a) Segmented iris image, (b) Red channel of the iris image, (c) Adaptive histogram equalization iris image

The patterns in the iris are not prominent, so in order to make them clearly visible, image enhancement is required. Adaptive histogram equalization is performed with a window size of $2 \mathrm{x}$ 2 at a clip limit of 0.01 , with full range and distribution, exponential to get the best result on the red channel of the iris image (as the iris patterns are most prominent in the red channel as shown in Figure $3 \mathrm{~b}$ ). The vessel structure becomes more prominent after the histogram equalization as shown in Figure $3 c$. The enhanced image is used further for feature extraction and identification. Sclera segmentation was performed by the fuzzy c-means method explained in [29].

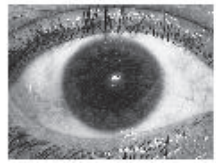

(a)

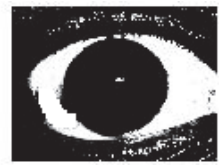

(b)
Figure 4: (a) grey image of 1(a). (b) shows the Fuzzy C means-based sclera segmentation of (a).

The vessels in the sclera are not prominent, so in order to make them clearly visible, image enhancement is required. Adaptive histogram equalization was performed with a window size of $42 \times 42$ in the green channel of the sclera image (as the sclera vessel patterns are most prominent in the green channel, which is shown in Figure 5(c)) to make the vessel structure more prominent as shown in Figure 6(a) and performed in [29]. Furthermore, the Discrete Meyer wavelet was used to enhance the vessel patterns. A low pass reconstruction of the abovementioned filter was used to enhance the image. Figure 6(b) shows the enhanced vessel image after applying the filter.

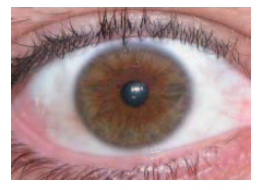

(a)

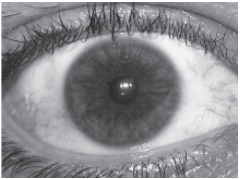

(c)

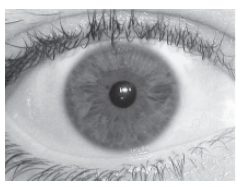

(b)

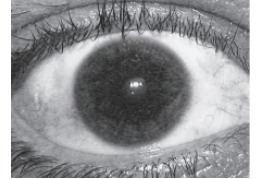

(d)
Figure 5: (a) The original RGB image, (b) The red channel component of (a), (c) The green channel component of (a), and (d) blue channel component of (a),

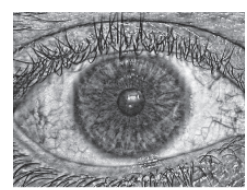

(a)

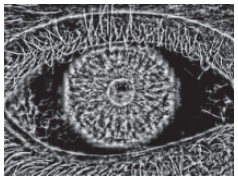

(b)
Figure 6: (a) Adaptive histogram equalization of the sclera image. (b) the vessel enhanced image after using a wavelet filter.

As proposed, the feature extraction approach uses images as an input therefore the image level fusion-based technique was used for combining both traits. 


\section{Results and discussion}

There are three parameters, $\mathrm{m}, \lambda$ and $\tau$ in the employed DPL model. To achieve the best performance, in our pattern recognition experiments, we set the number of dictionary atoms at their maximum (i.e. the number of training samples) for all competing DL algorithms, including the DPL.

In the recognition experiments, since the samples per class were 10, we set the number of dictionary atoms of each class as 10 for all the DL algorithms. Parameter $\tau$ is an algorithm parameter, and the regularization parameter $\lambda$ is to control the discriminative property of $\mathrm{P}$. In all the experiments, we choose $\lambda$ and $\tau$ by 10 -fold cross validation on the dataset explored. For all the competing methods, we tune their parameters for the best performance. The $\lambda$ and $\tau$ are set to 0.03 and 0.003 respectively. The $\gamma$ a bias factor is set as a small number: 0.0001 .

For experimental purposes, we have considered the traits individually as well as in combination, as well as their performance accuracy in percent, time complexity and with different image resolutions, which are given in Table 1.

For the above table it can be concluded that appreciable recognition performance accuracy is obtained by the trait using the proposed pattern classification scheme. It can also be confirmed from the above table that the time complexity of the system is also slightly dependent on the size of the image for the testing phase.

Furthermore, it can be observed from the above table that the images can work in low dimensions, containing less information; the difference in performance accuracy is also nearly negligible.

Table 1: Performance accuracy in percent, time complexity and with different image resolutions

\begin{tabular}{|c|c|c|c|c|}
\hline Traits & $\begin{array}{c}\text { Image } \\
\text { dimension }\end{array}$ & $\begin{array}{c}\text { Recognition } \\
\text { accuracy in } \\
\text { percentage }\end{array}$ & $\begin{array}{c}\text { Training } \\
\text { time } \\
\text { in } \\
\text { second }\end{array}$ & $\begin{array}{c}\text { Testing } \\
\text { time } \\
\text { in } \\
\text { second }\end{array}$ \\
\hline \multirow{2}{*}{ Iris } & $25 \times 25$ & 93.9 & 7.313 & 0.015 \\
\cline { 2 - 5 } & $50 \times 50$ & 94.12 & 103.440 & 1.056 \\
\cline { 2 - 5 } & $100 \times 100$ & 94.45 & 1000.589 & 1.09 \\
\hline \multirow{2}{*}{ Sclera } & $25 \times 50$ & 82 & 24.44 & 0.107 \\
\cline { 2 - 5 } & $50 \times 150$ & 82.99 & 560.23 & 1.1 \\
\cline { 2 - 5 } & $100 \times 200$ & 83.66 & 1560.33 & 1.12 \\
\hline \multirow{2}{*}{$\begin{array}{c}\text { Sclera } \\
\text { and iris }\end{array}$} & $25 \times 50$ & 97.3 & 24.44 & 0.107 \\
\cline { 2 - 5 } & $50 \times 150$ & 97.7 & 560.23 & 1.1 \\
\cline { 2 - 5 } & $100 \times 200$ & 97.99 & 1560.33 & 1.12 \\
\hline
\end{tabular}

The results of the proposed work are analysed with respect to the state-of-the-art by comparing it with the most similar work tested on the UBIRIS version 1 that could be found in the literature.

Table 2 reflects a state-of-the-art comparative analysis of the most similar work on the UBIRIS version 1 dataset.
Table 2: reflects a state-of-the-art comparative analysis of the most similar work on the UBIRIS version 1 dataset

\begin{tabular}{|c|c|c|c|c|}
\hline Work & Multisession & Multimodal & $\begin{array}{c}\text { Equal } \\
\text { Error } \\
\text { Rate } \\
\text { (in \%) }\end{array}$ & $\begin{array}{c}\text { Executio } \\
\text { n Time } \\
\text { in } \\
\text { second }\end{array}$ \\
\hline Zhou et al. [22] & No & Yes & 5.08 & \multirow{3}{*}{$\begin{array}{l}\text { Template } \\
\text { matching } \\
\text { so high }\end{array}$} \\
\hline Zhou et al. [25] & No & Yes & 2.73 & \\
\hline Das et al. [27] & No & No & 0.52 & \\
\hline Das et al. [28] & No & No & 0.66 & \multirow{3}{*}{$\begin{array}{c}\text { High } \\
\text { (SVM- } \\
\text { based } \\
\text { sparse } \\
\text { coding } \\
\text { DL) }\end{array}$} \\
\hline Das et al. [29] & Yes & No & 4.31 & \\
\hline Das et al. [30] & Yes & No & 3.95 & \\
\hline $\begin{array}{c}\text { Proposed } \\
\text { System }\end{array}$ & Yes & Yes & 2.01 & 1.12 \\
\hline
\end{tabular}

It can be confirmed from the above table that the proposed work has attained a higher recognition accuracy and lower time complexity than the state-of-the-art, which satisfies our hypothesis. From the table it can be seen that the method proposed by [27, 28] gives a slightly better result than our proposed method. The possible reason behind this fact is that in the authors' experiments, they have not considered a multisession experiment, and that is why they obtained better results than ours. In addition, our proposed method does not give better results than $[22,25]$. This is due to the fact that in very poor quality images (e.g. those with blur, blinking, or no sclera-area image), they could not be separated by a segmentation method so they were discarded in that study. However, in our proposed technique, it was able to segment most of the poor quality images, except those containing totally closed eyes, but they were also included in the proposed experiments (examples of such images are shown in Figure 2). Moreover, in their experiments, the authors have not considered a multisession experiment scenario.

In $[29,30]$ the research uses a multisession experiment scenario and the authors have also considered all images, but in contrast to our work, they have achieved a lower recognition accuracy.

With regards to the time complexity of the algorithm, our technique outperforms the systems proposed in [22, 25, 27], as they used template matching techniques. Whereas in $[28,29$, $30]$ dense patch-based features are used coupled with SPM and SVM and hence takes more than 2 seconds for classification of a query image. Whereas, our proposed algorithm could perform in $\sim 1$ second, preserving better accuracy. For a fair comparison we must also state that the reduction of the dimensionality can be assumed to be the reason behind the speed of our method. 
Although, it can be inferred from the above Table 2, that with the increase in dimensionality, this has impacted the time factor very negligibly. Furthermore, it is also important to mention that the image dimensionality would have hardly impacted the time performance of the systems proposed in [22, 25, 27]. The reason is because they used a patch-based feature, which is not dependent on the image size and thereby impacting the classification time. Therefore, from the aforementioned discussion it can be concluded that our proposed feature extraction technique performs more accurately and faster than any state-of-the-art techniques for eye biometrics in the visible spectrum. It is also worth mentioning that the proposed schemes have also proved to work well with lower dimensional images.

\section{CONCLUSIONS}

The present work proposes a projective pairwise dictionary learning-based approach for fast and efficient multimodal eye biometrics. The work uses Projective pairwise Discriminative DL in contrast to the traditional DL, which uses synthesis DL. Projective Pairwise Discriminative Dictionary uses a synthesis dictionary and an analysis dictionary jointly to achieve the goal of pattern representation and discrimination. As this DL is in contrast to the use of $1_{0}$ or $1_{1}$-norm sparsity constraints on the representation coefficients adopted in most traditional DLs, thus it works faster than other DLs. Moreover, the blending of the synthesis dictionary and an analysis dictionary also enhances the feature representation of the complex eye patterns. We employed the combination of sclera and iris traits from the established multimodal biometrics. The experimental study and analysis, which was conducted, fulfilled the hypothesis we considered. In this work we employed a part of the UBIRIS version 1 dataset to conduct the experiments. It can also be concluded that our proposed feature extraction technique performs more accurately and faster than any of the state-ofthe-art techniques for eye biometrics in the visible spectrum. It is also worth mentioning that the proposed schemes have also proved to work well with lower dimensional images, achieving appreciable recognition accuracy.

Future work will concentrate on using the proposed method for other traits.

\section{REFERENCES}

[1] J. Daugman, High Confidence visual recognition of persons by a test of statistical independence, IEEE Trans. Pattern Anal. Mach. Intell., 15(11) 1148-1160, 1993.

[2] R.P. Wildes, Iris recognition: An emerging biometric technology, Proc. IEEE, 85( 9), 1348-1363 1997.

[3] L. Masek, Recognition of human iris patterns for biometric identification, Master's thesis, University of Western Australia, 2003.

[4] D. Lowe, Distinctive image features from scale-invariant key points," Intl Journal of Computer Vision, 60(2), 91-110, 2004.

[5] C. Rathgeb and A. Uhl, Secure iris recognition based on local intensity variations, in Proc ICIAR, 6112, 266-275, 2010.

[6] D. Monro, S. Rakshit, and D. Zhang, DCT-Based iris recognition, IEEE Trans. PAMI, 29( 4), 586-595, 2007.
[7] J.-G. Ko, Y.-H. Gil, J.-H. Yoo, K.-I. Chung, A novel and efficient feature extraction method for iris recognition, ETRI Journal, 29(3), 399-401, 2007.

[8] L. Ma, T. Tan, Y. Wang, D. Zhang, Personal identification based on iris texture analysis, IEEE Tans. PAMI, 25( 2), 1519-1533, 2003.

[9] J. Wright, A.Y. Yang, A. Ganesh, S. S. Sastry, Y. Ma, Robust face recognition via sparse representation, IEEE Trans. PAMI , 31(2), 210-227, 2009.

[10] J. Mairal, F. Bach, J. Ponce, Task-driven dictionary learning, IEEE Trans PAMI , 34(4), 791-804, 2012

[11] Z. Jiang, Z. Lin, L. Davis, Label consistent k-svd: learning a discriminative dictionary for recognition, IEEE Trans. PAMI 35(11), 2651-2664, 2013

[12] M. Aharon, M. Elad, A. Bruckstein, K-svd: An algorithm for designing overcomplete dictionaries for sparse representation, IEEE Trans. on Signal Processing, 54(11), 4311-4322, 2006

[13] S. Gu, L. Zhang, W. Zuo, X. Feng., Projective dictionary pair learning for pattern classification., In NIPS 2014, 1-9, 2014..

[14] J. Mairal, F. Bach, J. Ponce, G. Sapiro, A. Zisserman, "Supervised dictionary learning," In: NIPS., 1033-1040, 2008

[15] I. Ramirez, P. Sprechmann, G. Sapiro, Classification and clustering via dictionary learning with structured incoherence and shared features, In: CVPR, 3501-3508, 2010

[16] M. Yang, L. Zhang, X. Feng, D. Zhang, Fisher discrimination dictionary learning for sparse representation, In: ICCV, 543-550, 2011

[17] Z. Wang, J. Yang, N. Nasrabadi, T. Huang, A max-margin perspective on sparse representation-based classification, In: ICCV., 1217-1224, 2013

[18] M. Soltanolkotabi, E. Elhamifar, E. Candes, Robust subspace clustering, The Annals of Statistics, 42(2), 669-699, 2014.

[19] A. Coates, A.Y. Ng, The importance of encoding versus training with sparse coding and vector quantization, In: ICML. 921-928, 2011

[20] L. Zhang, M. Yang, X. Feng, Sparse representation or collaborative representation: Which helps face recognition?, In: ICCV., 471-478, 2011

[21]R. Derakhshani, A. Ross, and S. Crihalmeanu. A new biometric modality based on conjunctival vasculature. Proceedings of Artificial Neural Networks in Engineering: 1-8, 2006.

[22]Z. Zhou, Y. Du, N. L. Thomas, and E. J. Delp. Multimodal eye recognition. Proceedings of the International Society for Optical Engineering, 7708(770806):1-10, 2010.

[23]Z. Zhou, Y. Du, N. L. Thomas, and E. J. Delp. A new biometric sclera recognition. IEEE transaction on System, Man And Cybernatics -PART A: System And Human, 42(3): 571-583, 2012.

[24]Z. Zhou, Y. Du, N. L. Thomas, and E. J. Delp, Quality Fusion Based Multimodal Eye Recognition, IEEE International Conference on Systems, Man, and Cybernetics, 1297-1302, 2012.

[25] V. Gottemukkula, S. K. Saripalle, S. P. Tankasala, R. Derakhshani, R. Pasula and A. Ross, Fusing Iris and Conjunctival Vasculature: Ocular Biometrics in the Visible Spectrum, IEEE Conference on Technologies for Homeland Security: 150-155, 2012.

[26] A. Das, U. Pal, M. Blumenstein and M. A. Ferrer, Sclera Recognition - A Survey, Recent Advancement in Computer Vision and Pattern Recognition, 917 -921, 2013.

[27] A. Das, U. Pal, M. A. Ferrer and M. Blumenstein, A New Method for Sclera Vessel Recognition using OLBP, Chinese Conference on Biometric Recognition ,LNCS 8232, . 370-377,2013.

[28] A. Das, U. Pal, M. A. Ferrer and, M. Blumenstein, Sclera Recognition Using D-SIFT, In 13th International Conference on Intelligent Systems Design and Applications: 74-79, 2013.

[29] A. Das, U. Pal, M. A. Ferrer and, M. Blumenstein, Fuzzy Logic Based Sclera Recognition, In FUZZ-IEEE: 561-568, 2014.

[30] A. Das, U. Pal, M. A. Ferrer and M. Blumenstein, A New Efficient and Adaptive Sclera Recognition System, IEEE Symposium Series on Computational Intelligence, 1-6, 2014.

[31] A. Das, U. Pal, M. A. Ferrer and M. Blumenstein, Multi-angle Based Lively Sclera Biometrics at a Distance, IEEE Symposium Series on Computational Intelligence, 22-29, 2014..

[32] A. Das, R. Kunwar, U. Pal, M. A. Ferrer, M. Blumenstein, An Online Learning-Based Adaptive Biometric System, A Book on Adaptive Biometric Systems, 73-96, 2015.

[33] H. Proença and L. A.Alexandre, UBIRIS: A noisy iris image database, Proceed. of ICIAP 2005 - Intern. Confer. on Image Analysis and Processing, 1, 970-977,2005. 